\title{
Adrenal Myelolipoma with Haemorrhage
}

\author{
Ranjan Kumar ${ }^{1 *}$, Manish Kumar Choudhary ${ }^{2}$ and Shorav Bhatnagar ${ }^{3}$ \\ 'Department of Pathology, QRG Central Hospital \& research Centre, Faridabad, Haryana, INDIA \\ ${ }^{2}$ Department of Urology, QRG Health City, Faridabad, Haryana, INDIA \\ ${ }^{3}$ Department of Radio diagnosis \& Imaging, QRG Central Hospital \& Research Centre, Faridabad, Haryana, INDIA
}

\section{ABSTRACT}

Myelolipomas are a rare benign variant of adipose tissue tumors with additional hematopoietic elements. Generally, these tumors were discovered accidentally on autopsy with a low incidence. Adrenal gland is the most common site for myelolipoma. We report a case of adrenal myelolipoma $(7 \mathrm{~cm})$ in female in her 4th decade who presented with pain right side abdomen which can be confused with other adrenal malignancy.

\section{Keywords: Myelolipoma, Adrenal}

\section{Introduction}

Adrenal myelolipomas are unusual, benign tumors of adrenal gland that are usually asymptomatic. There has been an increase in detection of these lesions due to more frequent use of imaging techniques. ${ }^{[1]}$ Majority of these tumors are diagnosed incidentally although some may present with varied symptoms ${ }^{[2]}$. Histologically, they are composed of an admixture of lipomatous component and hematopoietic component, in varying proportions. We report a case of adrenal myelolipoma, in a woman who presented with right abdomen pain for 2 months. Patient was treated surgically by Laparoscopic removal of tumor

\section{Case Report}

A 47 year old women presented with complain of headache, nausea, pain in neck region for 2-3 months and right abdomen pain. She had history of hypothyroidism. Relevant investigations were done which included NonContrast CT head, Chest X ray PA, X ray cervical Spine and Ultrasound Whole abdomen. Hematological investigation revealed mildly raised ESR, raised AST, ALT. Ultrasound revealed gall bldder stone with a well-defined echogenic area measuring $7.4 \times 5.9 \mathrm{~cm}$ in right suprarenal area and CT KUB with Contrast was advised for further workup. CT KUB with contrast showed mixed attenuating space occupying lesion in right supra renal gland with foci of calcification without any enhancement on contrast study and finding suggesting of? Lipid rich adenoma was given

The patient was admitted for Laparoscopic cholecystectomy and right adrenalectomy. Systemic examination revealed Blood pressure of 120/70 $\mathrm{mm} \mathrm{Hg}$, Respiratory Rate $20 / \mathrm{min}$, Temperature 98.6F and Spo2 98\%. The patient underwent Laparoscopic right adrenalectomy and cholecystectomy and his postoperative recovery period was uneventful.

\section{Pathological Examination}

Gross examination shows a well circumscribed capsulated greyish yellow soft tissue piece measuring $7 \mathrm{~cm} \times 7 \mathrm{~cm}$ was received in pathology laboratory for histopathological examination [Figure 1]. On serial sectioning, variegated appearance was noted from gray-yellow to gray-brown with extensive hemorrhagic [Figure 2]. Microscopic Examination showed a well encapsulated tissue showing extensive area of mature adipose tissue with admixed hematopoietic elements comprising predominantly megakaryocytes, erythroid cells and myeloid cells with compressed adrenal tissue adjacent to capsule. There is presence of hemorrhagic areas[Figure 3]. The Histopathological examination confirmed Adrenal Myelolipoma

\section{Discussion}

Adrenal myelolipoma was first described by Giercke in 1905, but the term "myelolipoma" was coined by Oberlin, some 24 years later in $1929 .^{[3,4]}$. It is a lesion in the adrenal gland composed of variable amounts of mature adipose tissue and all three hematopoietic lineages of the bone marrow, i.e., white blood cells, red blood cells, and megakaryocytes ${ }^{[5]}$. However, it does not serve as a hematopoietic source. ${ }^{[5]}$ Most are small $(<5 \mathrm{~cm})$, but giant myelolipomas, although rare, have also been reported. In our case the size of tumor was $7 \mathrm{~cm}$

Many hypotheses have been put forward to explain the etiology of myelolipomas, yet it is unclear. According to a new theory, it is believed that adrenal myelolipoma originates from metaplasia of reticuloendothelial cells in adrenal capillaries or from undifferentiated stromal cells in the zona fasciculate of the adrenal cortex whereas some 


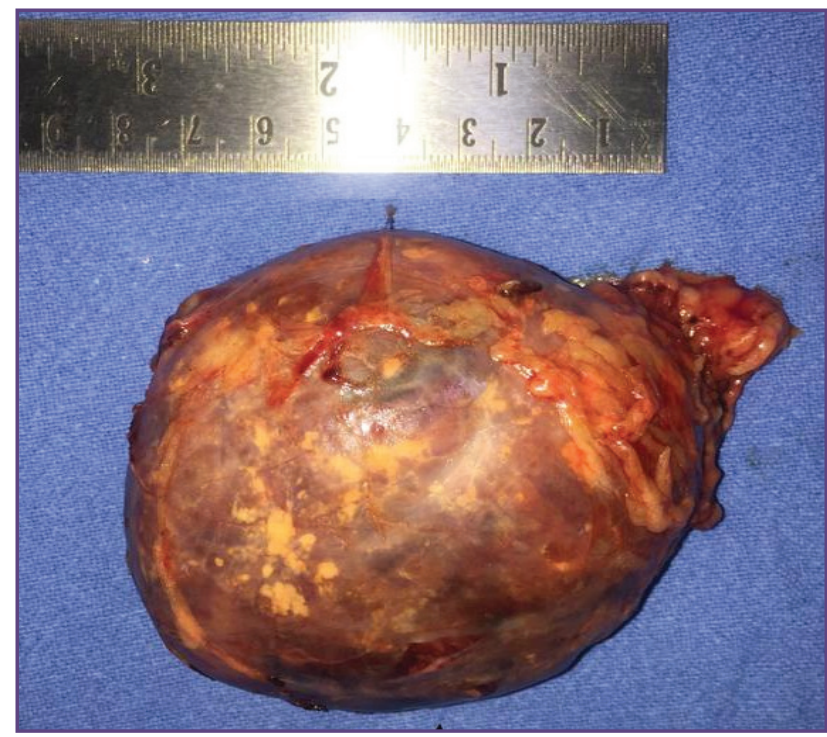

Fig. 1: Gross Appearanc of Adrenal Mass.

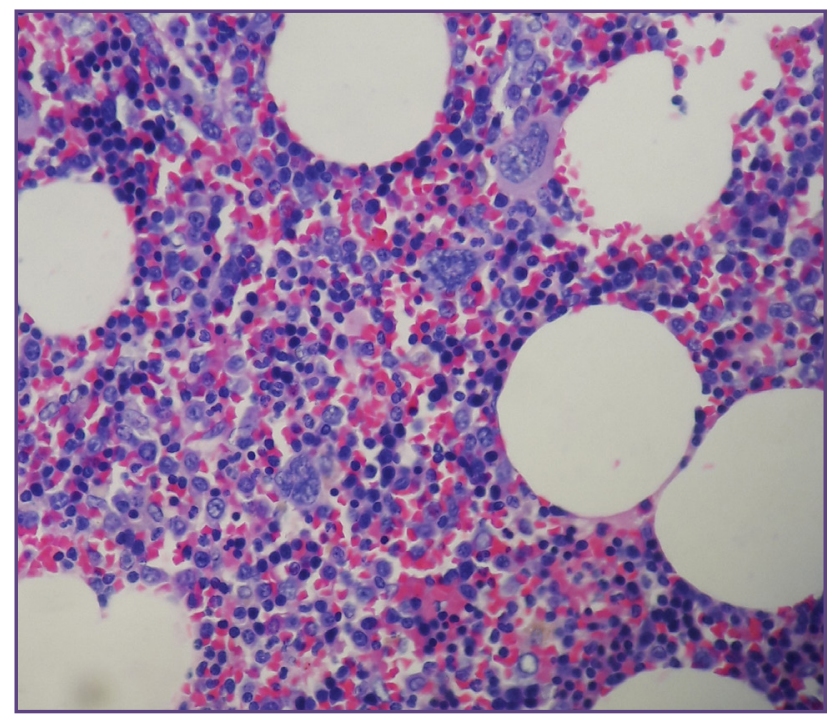

Fig. 3 : H\&E section of adrenal mass showing hematopoietic elements and adipose tissue(100X).

believe that these tumors arise from embryonic cells of bone marrow that embolize to adrenal gland. However, some authors believe it to be the result of endocrine stimulation, stressful and unbalanced lifestyle, and chronic systemic diseases. They can also be found in association with obesity, hypertension, and a variety of chronic diseases ${ }^{[6]}$ In our case patient was known case of hypothyroidism

Recently Laparoscopic adrenalectomy has been shown to be an effective methodology for excision of adrenal myelolipoma with lesser blood and short duration of postoperative day ${ }^{[7]}$. In our case patient underwent laparoscopic adrenalectomy and it was uneventful

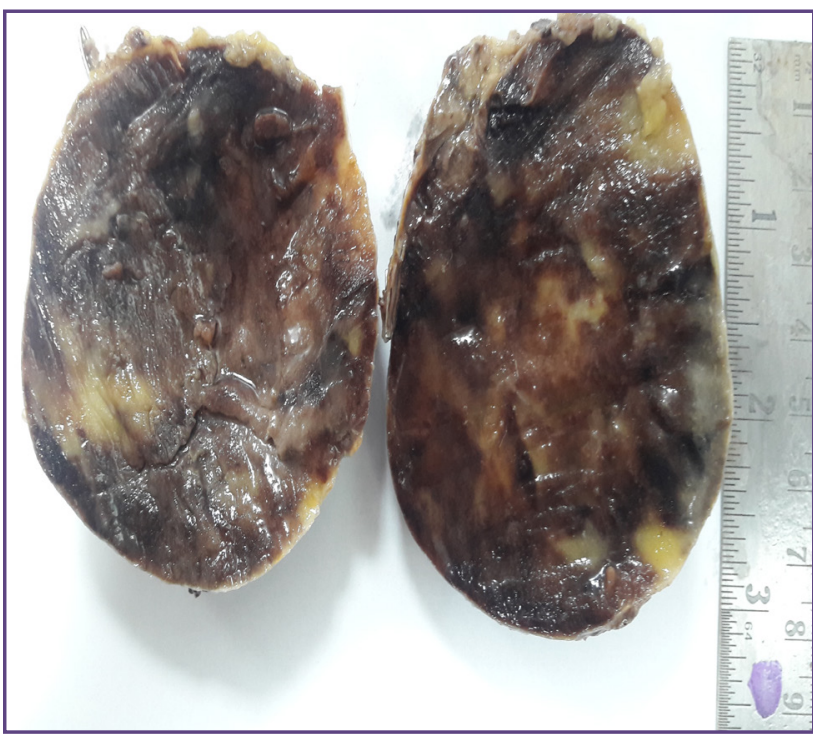

Fig. 2 : On cut section reddish brown to yellowish area seen.

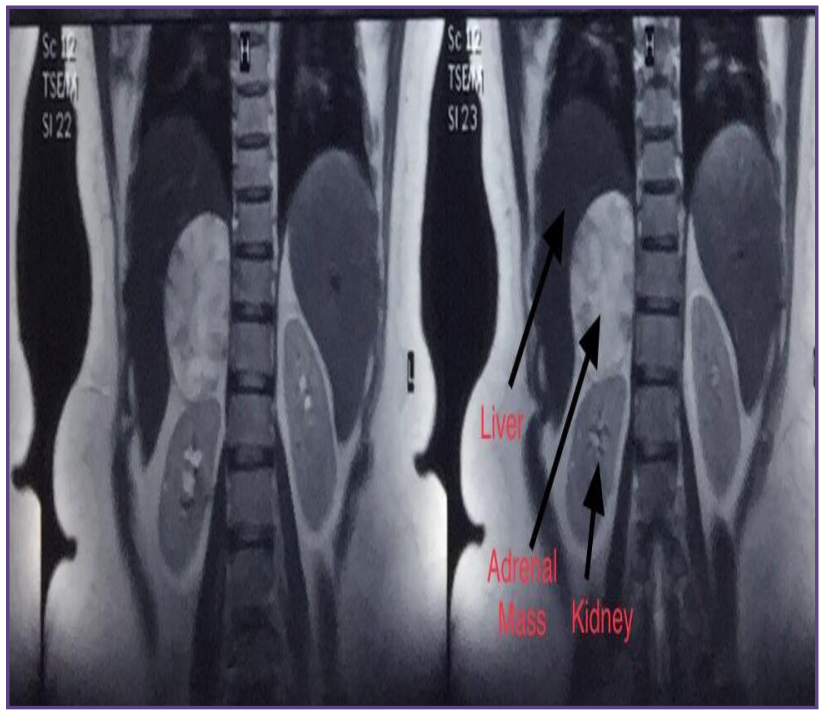

Fig. 4: CT image showing adrenal tumor.

Diagnostic modalities such as ultrasonography, CT, and magnetic resonance imaging are effective in detecting more than $90 \%$ of adrenal myelolipomas ${ }^{[8]}$; CT was most sensitive as it can identify fat in the adrenal gland. ${ }^{[8]}$ On CT imaging, myelolipomas are seen as heterogeneous masses with low-density mature fat interspersed with more dense myeloid tissue. ${ }^{[9]}$.As in our case ultrasound detected well defined echogenic area and Contrast CT detected the lesion and finding were suggestive of Lipid rich adenoma

\section{Conclusion}

Adrenal myelolipoma are rare, benign adrenal lesion that are usually detected incidentally on imaging studies 
.CT is very sensitive in detecting these lesion due to lipid content of tumor. Proper workup should be done as lesion is benign and surgical excision is recommended to prevent complication in large tumors. Laparoscopic surgery is very effective with good patient outcome and minimal complication as done in our case successfully. Adrenal Myelolipoma should also be considered as differential diagnosis as it has good prognosis \& out come and small lesion do not require surgery and in case of large tumor it can be easily removed laparoscopically

\section{Abbreviations \& Symbols}

CT- Computed tomography, KUB-Kidney ureter bladder, ESR-erythrocyte sedimentation rate

\section{Acknowledgements}

Nil

\section{FUNDING}

Nil

\section{Competing Interests}

There are no conflicts of interest

\section{Reference}

1. Shenoy VG, Thota A, Shankar R, Desai MG. Adrenal myelolipoma: Controversies in its management. Indian J Urol. 2015. Apr-Jun; 31(2): 94-101.2. Gershuni VM, Bittner JG.
2. Ivth, Moley JF, Brunt LM. Adrenal Myelolipoma: Operative Indications and Outcomes. J Laparoendosc Adv Surg Tech A. 2014 Jan;24(1);8-12.

3. Sajjanar AB, Athanikar VS, Dinesh US, Nanjappa B, Patil PB. Non functional unilateral adrenal myelolipoma, a case report. J Clin Diagn Res. 2015;9:ED03-4.

4. Kenney PJ, Wagner BJ, Rao P, Heffess CS. Myelolipoma: CT and pathologic features. Radiology. 1998;208:87-95.

5. Barman S, Mandal KC, Mukhopadhyay M. Adrenal myelolipoma: An incidental and rare benign tumor in children. J Indian Assoc Pediatr Surg. 2014;19:236-8.

6. Dellaportas D, Tsagkas A, Myoteri D, Contis J, Pafiti AK. Clinicopathological characteristics of our cases of adrenal myelolipomas: A rare surgical entity. World J Oncol 2012;3:294-7

7. Suguru Yamashita, Kei Ito, Kaoru Furushima, Junichi Fukushima, Shuji Kameyama, Yasushi Harihara Laparoscopic versus open adrenalectomy for adrenal myelolipoma Ann Med Surg (Lond) 2014 Jun; 3(2): 34-38.

8. Sajjanar AB, Athanikar VS, Dinesh US, Nanjappa B, Patil PB. Non functional unilateral adrenal myelolipoma, a case report. J Clin Diagn Res. 2015;9:ED03-4.

9. Kale G, Pelley EM, Davis DB. Giant myelolipomas and inadvertent bilateral adrenalectomy in classic congenital adrenal hyperplasia. Endocrinol Diabetes Metab Case Rep. 2015;2015:150079.

*Corresponding author:

Dr. Ranjan Kumar, QRG central Hospital \& Reasearch Centre, Near Neelam Flyover, Ajronda chowk, Faridabad , Haryana, 121002 , INDIA

Phone: +91 9971667753

Email: drkumar.ranjan@gmail.com

Financial or other Competing Interests: None. 